\title{
Estructura de tallas y crecimiento de los cangrejos Callinectes arcuatus y C. bellicosus (Decapoda: Portunidae) en la laguna costera Las Guásimas, México
}

\author{
Luis Hernández \& José Alfredo Arreola-Lizárraga \\ Centro de Investigaciones Biológicas del Noroeste, S.C. (CIBNOR) Unidad Sonora Carr. a Las Tinajas km 2.3 Predio \\ El Tular, A.P. 349, C.P. 85454, Guaymas, Sonora, México. Fax: + 62222 122 37; aarreola04@cibnor.mx
}

Recibido 06-VI-2003. Corregido 08-VI-2006. Aceptado 15-XII-2006.

\begin{abstract}
Size frequency distribution and growth of the crabs Callinectes arcuatus and C. bellicosus (Decapoda: Portunidae) in Las Guásimas coastal lagoon, Mexico. Information on size frequency distribution, the width-weight relation and growth parameters of the crabs Callinectes arcuatus and C. bellicosus is presented. The data comes from samples taken with a trawl net both day and night on a monthly basis from March 1998 to February 2000 in a coastal lagoon from Gulf of California. C. bellicosus $(\mathrm{n}=878)$ was more abundant than $C$. arcuatus $(\mathrm{n}=357)$ and its size frequency distribution presented carapace width $\mathrm{CW}$ ranges of 8.4-166 mm and 9-130 $\mathrm{mm}$ respectively. Both populations were mainly represented by juveniles $(75 \mathrm{~mm}$ in $\mathrm{CW})$ ranging from 37 to $75 \mathrm{~mm}$ in $\mathrm{CW}$, and adults $(>75 \mathrm{~mm}$ in $\mathrm{CW}$ ) between 76 and $90 \mathrm{~mm}$ in CW. In both species the widthweight relation showed that males grow more than females, with an isometric growth tendency being observed. The growth parameters for $C$. arcuatus estimated using the von Bertalanffy Model, were: $\mathrm{K}=0.84$ year ${ }^{-1}$, $\mathrm{L}_{\infty}=$ $140 \mathrm{~mm}$ to $=-0.12$ for $C$. arcuatus, and $\mathrm{K}=0.9$ year $^{-1}, \mathrm{~L} \infty=169 \mathrm{~mm}$ to $=-0.11$ for $C$. bellicosus. These results showed that the relative age at which maximum growth is attained is between three and four years for both species. Rev. Biol. Trop. 55 (1): 225-233. Epub 2007 March. 31.
\end{abstract}

Key words: swimming crabs, growth, size frecuency, Callinectes arcuatus, Callinectes bellicosus.

Las jaibas Callinectes arcuatus (Ordway, 1863) y Callinectes bellicosus (Stimpson, 1859) habitan la costa del océano Pacífico desde el sur de California, EEUU. hasta Colombia y Golfo de Tehuantepec México, respectivamente (Brusca 1980, Hendrickx 1995). Estas especies, como las otras del género Callinectes, presentan una fase planctónica en mar abierto para el desarrollo de la larva y una fase bentónica en lagunas costeras y estuarios para su crecimiento y reproducción (Williams 1974). Observaciones en el golfo de California mostraron que $C$. arcuatus es una especie eurihalina que tolera un intervalo de salinidad de 1-65\%o, se encuentra en un intervalo de temperatura de $17.5-34{ }^{\circ} \mathrm{C}$ y habita en profundidades de $0-40 \mathrm{~m}$; mientras que $C$. bellicosus es una especie estenohalina que tolera un intervalo de salinidad de 31-38 $\%$, se encuentra en un intervalo de temperatura de $18-4{ }^{\circ} \mathrm{C}$ y habita en profundidades de 0-30 m (Paul 1982a).

Arreola-Lizárraga et al. (2003) presentaron un modelo conceptual acerca de la ecología de $C$. arcuatus y $C$. bellicosus en lagunas semiáridas subtropicales del golfo de California, sugiriendo que durante primavera y verano es la temporada reproductiva con la presencia de jóvenes y adultos, así como machos y hembras en el interior de las lagunas; durante otoño la abundancia es la más baja y únicamente ocurren machos adultos en las inmediaciones de las bocas de las lagunas; durante invierno ocurre el principal reclutamiento de jóvenes, 
los cuales se concentran principalmente en las porciones más internas de las lagunas.

Por otro lado, ambas especies constituyen un recurso pesquero importante debido a la fuente alimenticia y el empleo. En México, en el 2001 la pesquería tuvo un valor de $\$ 166786$ 000.00 y la producción fue de 18495 ton peso vivo, de las cuales 11204 ton correspondieron al litoral Pacífico y 7291 ton al golfo de México y mar Caribe; en el Pacífico destaca la producción pesquera del golfo de California aportada por los estados de Sonora (4 478 ton) y Sinaloa (4 685 ton) (Ramírez-Félix et al. 2003). En este sentido, el conocimiento de la estructura de tallas y del crecimiento de los individuos es relevante porque proporciona información de la dinámica local de la población estudiada y es posible establecer tallas de captura, que garanticen que la pesca está incidiendo sobre adultos post reproductivos para permitir que los jóvenes puedan llegar a la edad reproductiva y después integrarse al stock explotado.

Estudios sobre estructura de tallas y crecimiento de C. arcuatus (Paul 1982b, Dittel y Epifanio 1984, Nevárez-Martínez et al. 2003) y C. bellicosus (Molina-Ocampo 2001, NevárezMartínez et al. 2003) han sido realizados en localidades del Pacífico. El presente estudio contribuye con información de tallas y crecimiento a partir de jaibas capturadas en la laguna costera Las Guásimas, una laguna semiárida subtropical, que representa una las localidades pesqueras tradicionales de este recurso en el golfo de California. Los objetivos fueron: (1) conocer la estructura de tallas, (2) estimar la relación ancho-peso y (3) determinar los parámetros de crecimiento para ambas especies de jaibas.

\section{MATERIALES Y MÉTODOS}

La laguna costera Las Guásimas se localiza en la costa este del golfo de California $\left(27^{\circ} 49^{\prime}-55^{\prime} \mathrm{N}, 110^{\circ} 29^{\prime}-45^{\prime} \mathrm{W}\right)$, tiene una superficie de $37 \mathrm{~km}^{2}$, con profundidad promedio de $0.7 \mathrm{~m}$ y una boca de $2 \mathrm{~km}$ de largo que la comunica permanentemente con el golfo de
California (Arreola-Lizárraga et al. 2003). Con base en los criterios de clasificación de Kjerfve y Magill (1989), es una laguna del tipo "restringida", considerando que está comunicada permanentemente con el mar a través de una boca, tiene una circulación por mareas bien definida, es influenciada fuertemente por vientos y es bien mezclada verticalmente.

Las jaibas fueron recolectadas durante el día y la noche mensualmente desde marzo 1998 hasta febrero 2000 en seis estaciones de muestreo que se ubicaron siguiendo los canales de la laguna (Fig. 1). Los muestreos se realizaron con una red de arrastre tipo camaronera de $6 \mathrm{~m}$ de largo y $3 \mathrm{~m}$ de ancho con luz de malla de $2.5 \mathrm{~cm}$. En cada estación, los lances tuvieron una duración de 10 min y se registraron la temperatura y salinidad con equipo multisensor marca Hydrolab modelo Recorder. Las jaibas capturadas se colocaron en bolsas de plástico y se mantuvieron en hielo hasta su traslado al laboratorio donde se llevó a cabo la identificación y biometrías. La identificación de las especies se basó en la forma de las espinas frontales y el sexo se determinó por la forma del abdomen siguiendo los criterios de Rathbun (1930) y Hendrickx (1995). Las biometrías consistieron en medir el ancho del caparazón (AC) que es la distancia entre las puntas de las espinas anterolaterales con un vernier de $0.1 \mathrm{~mm}$ de precisión, enseguida los individuos fueron pesados en una balanza College 300 con $0.01 \mathrm{~g}$ de precisión.

Los parámetros de crecimiento K y L $\infty$ se calcularon con el paquete FISAT, el cual estima dos parámetros adicionales: C (amplitud de crecimiento) que determina la amplitud de las oscilaciones de la curva de crecimiento y WP (punto de invierno) que permite identificar el momento del año en que el crecimiento es más lento. Con estos parámetros se obtuvo la ecuación de crecimiento del modelo von Bertalanffy (Sparre y Venema 1995):

$$
\mathrm{Lt}=\mathrm{L} \infty\left[1-\mathrm{e}^{-\mathrm{K}(\mathrm{t}-\mathrm{to})-(\mathrm{CK} / 2 \pi) * \operatorname{sen}(2 \pi(\mathrm{t}-\mathrm{ts})}\right]
$$

donde:

$\mathrm{Lt}=$ Ancho del caparazón (AC) al tiempo $\mathrm{t}$ 


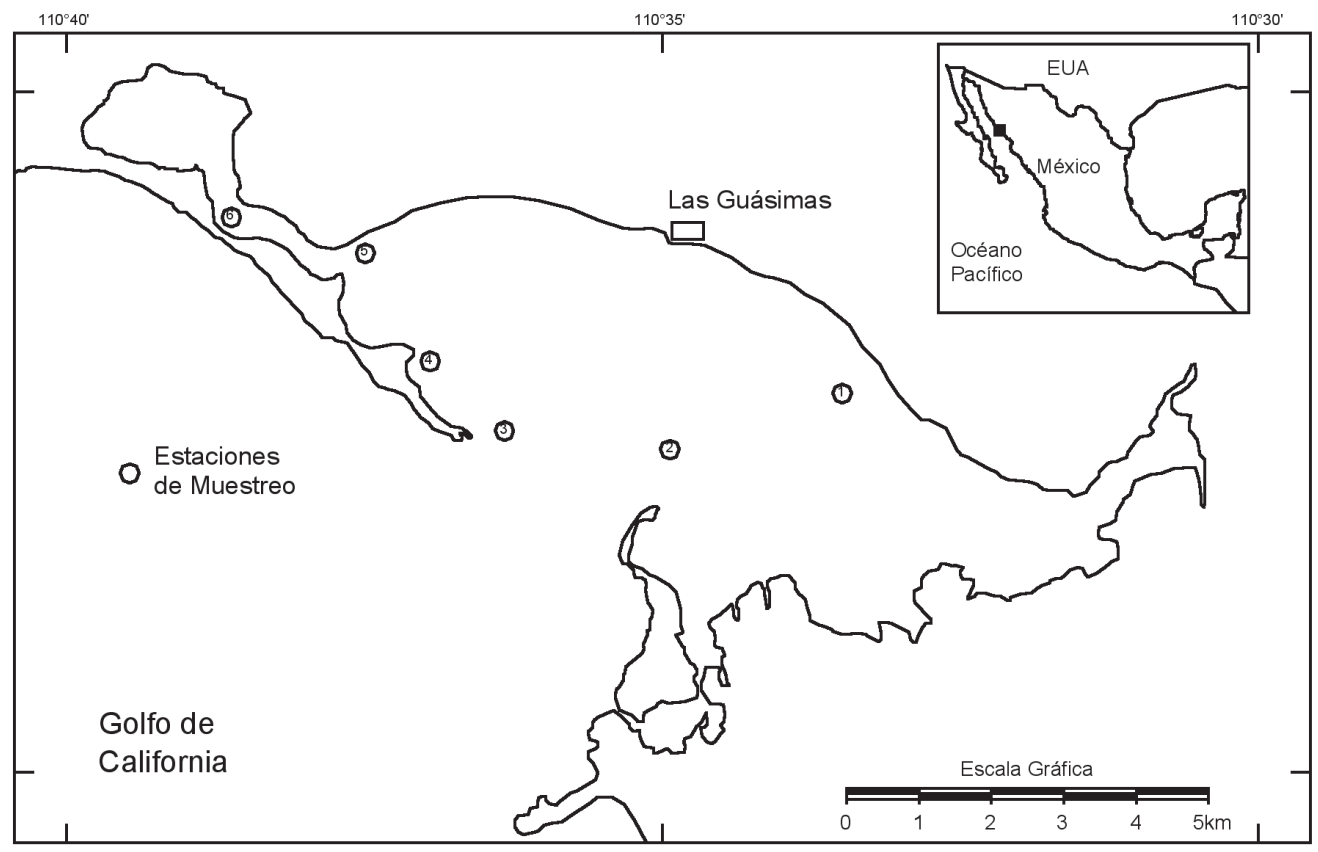

Fig. 1. Área de estudio y estaciones de muestreo.

Fig. 1. Study area and sampling stations.

$\mathrm{L} \infty=$ Ancho asintótico del organismo

$\mathrm{K}=$ Coeficiente de crecimiento de von Bertalanffy

$\mathrm{C}=$ Amplitud de crecimiento

$\mathrm{WP}=$ punto de invierno $(=\mathrm{ts}+0.5)$ tiempo en el que el crecimiento es mínimo

$\pi=3.141592 \ldots$

$\mathrm{Ts}=$ Punto de verano $(=$ tasa de crecimiento máximo)

to $=$ Teóricamente es el tiempo en el que el organismo tiene talla cero, y se calculó por la ecuación empírica de Pauly (1980):

$$
\begin{aligned}
\log -t_{0}= & -0.3922-(2752 \log \mathrm{L} \infty) \\
& -(1.038 \log \mathrm{K})
\end{aligned}
$$

La relación ancho-peso se calculó aplicando el modelo potencial (Pauly 1980):

$\mathrm{W}=\mathrm{a}(\mathrm{AC})^{\mathrm{b}}$

donde:

$\mathrm{W}=$ peso $(\mathrm{g})$

A.c. $=$ Ancho del caparazón $(\mathrm{mm})$

a y $b=$ Parámetros de la regresión
El criterio para separar jóvenes y adultos se obtuvo mediante un gráfico del número de hembras ovígeras contra los intervalos de tallas.

Los parámetros de crecimiento estimados en este estudio se compararon con resultados en contribuciones previas mediante el índice de la Fi prima de Munro $\varnothing^{\prime}=\log (\mathrm{k})+2 *$ $\log (\mathrm{L} \infty)$ que representa la eficiencia del crecimiento (Munro y Pauly 1983).

\section{RESULTADOS}

En la laguna costera Las Guásimas, la temperatura del agua tuvo un intervalo anual de $17-34{ }^{\circ} \mathrm{C}$ con valores de $30-34{ }^{\circ} \mathrm{C}$ en verano (junio-septiembre) y de $17-20^{\circ} \mathrm{C}$ en invierno (diciembre-marzo). La salinidad tuvo un intervalo anual de 37-41 \% y no mostró un patrón estacional definido.

Se capturaron 1235 individuos, de los cuales 357 fueron de C. arcuatus (193 machos y 164 hembras) y 878 fueron de C. bellicosus (393 machos y 485 hembras). 

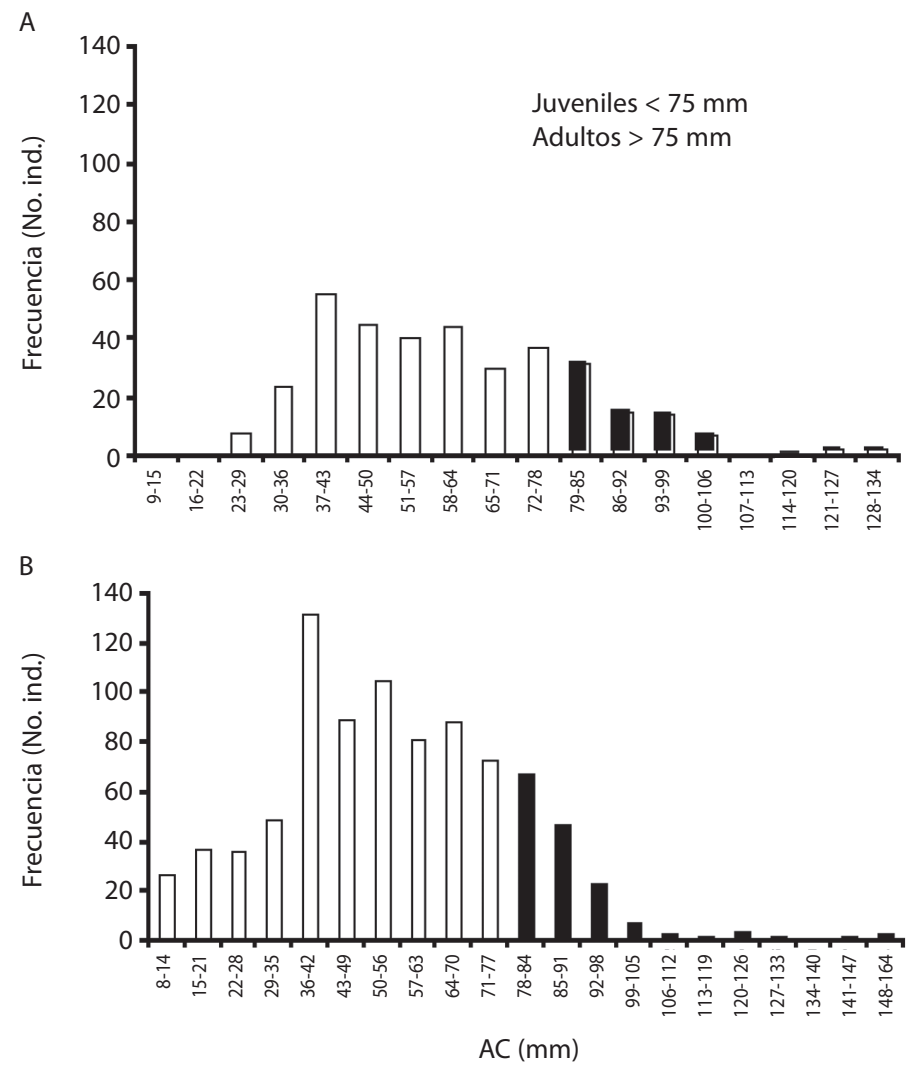

Fig. 2. Estructura de tallas: A. C. arcuatus y B. C. bellicosus.

Fig. 2. Size structure: A. C. arcuatus and B. C. bellicosus.

Se obtuvieron 22 hembras ovígeras de $C$. arcuatus con un intervalo de talla de 54-122 $\mathrm{mm} \mathrm{AC}$, el $50 \%$ de ellas fue un ejemplar de $73 \mathrm{~mm}$ AC por lo que a partir de $74 \mathrm{~mm} \mathrm{AC} \mathrm{se}$ consideraron individuos adultos. Se obtuvieron 60 hembras ovígeras de $C$. bellicosus con un intervalo de talla de 51-95 mm AC, el $50 \%$ de ellas fue un ejemplar de $74 \mathrm{~mm}$ AC por lo que a partir de $75 \mathrm{~mm}$ AC se consideraron individuos adultos. Para fines prácticos se consideró que los individuos de ambas especies menores a 75 $\mathrm{mm}$ AC son jóvenes y los individuos mayores a $75 \mathrm{~mm}$ AC son adultos.

Los individuos de C. arcuatus tuvieron un intervalo de tallas de 9-30 mm AC, los jóvenes representaron el $80 \%$ y los adultos el $20 \%$ (Fig. 2). Los individuos de C. bellicosus tuvieron un intervalo de tallas de 8.4-66 mm AC, los jóvenes representaron el $82 \%$ y los adultos el $18 \%$ (Fig. 2).

La relación ancho-peso mostró que los machos crecen más que las hembras en ambas especies y se observó una tendencia de crecimiento isométrico en todos los casos (Fig. 3, 4).

Los parámetros de crecimiento estimados del modelo von Bertalanffy para $C$. arcuatus fueron: $\mathrm{K}=0.84$ año $^{-1}, \mathrm{~L} \infty=140$ a $-0.12 \mathrm{~mm}$, y para $C$. bellicosus fueron: $\mathrm{K}=0.9$ año $^{-1}, \mathrm{~L} \infty=$ 169 a $-0.11 \mathrm{~mm}$. Estos resultados mostraron que la edad relativa a la cual se alcanza el crecimiento máximo es entre tres y cuatro años para ambas especies (Fig. 5). En el modelo no se registraron efectos de la temperatura sobre los parámetros de crecimiento para ninguna de las especies estudiadas, por lo tanto el punto de invierno y la amplitud de la curva de 

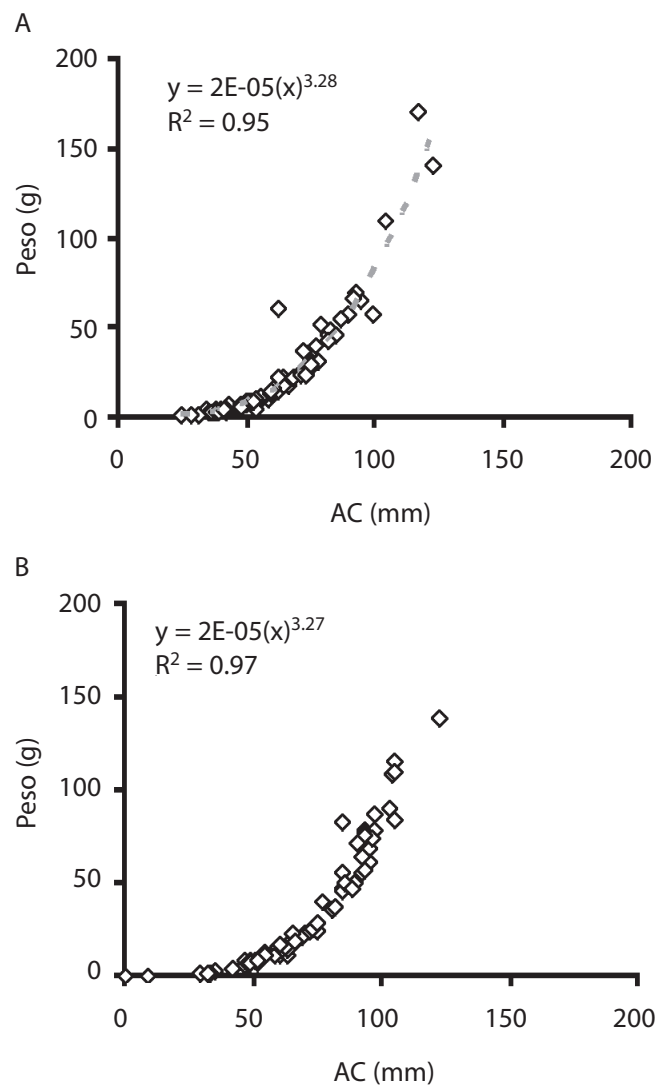

Fig. 3 Relación entre el ancho del caparazón y el peso total de C. arcuatus: A. machos, B. hembras.

Fig. 3. Relationship between the carapace width and total weight of C. arcuatus: A. males, B. females.

crecimiento no fueron detectados en el modelo $(\mathrm{WP}=0 ; \mathrm{C}=0)$.

\section{DISCUSIÓN}

En la laguna costera Las Guásimas la variabilidad anual de la salinidad (35-41\%o) es un factor clave en la dominancia de $C$. bellicosus sobre $C$. arcuatus 2.4:1 (ArreolaLizárraga et al. 2003); estos autores consideran las observaciones Paul (1982a), en el sentido de que $C$. bellicosus es estenohalina con desarrollo óptimo en salinidades de 30-38 $\%$; mientras que $C$. arcuatus aunque es eurihalina (1-65\%), presenta mejor desarrollo en
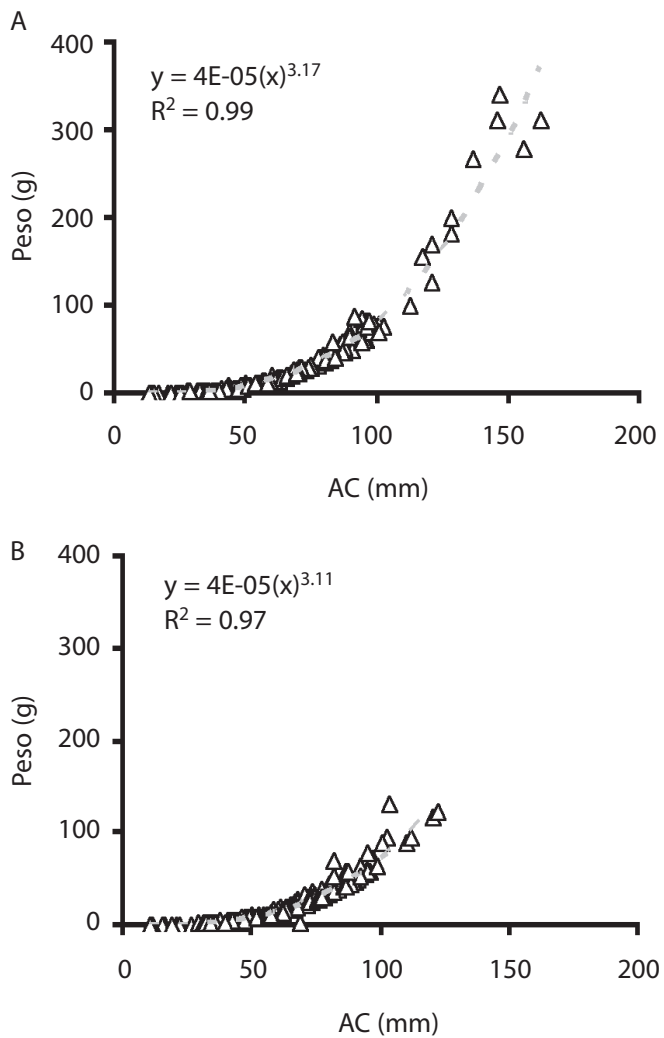

Fig. 4. Relación entre el ancho del caparazón y el peso total de C. bellicosus: A. machos, B. hembras.

Fig. 4. Relationship between the carapace width and total weight of $C$. bellicosus: A. males, B. females.

lagunas costeras cuya salinidad disminuye por debajo de $30 \%$.

Estructura de tallas: La población de $C$. arcuatus se compuso por $80 \%$ de jóvenes $(<75$ mm AC), el $82 \%$ de los adultos tuvieron tallas entre 51 y 85 mm AC (Fig. 2); mientras que la población C. bellicosus se compuso por $82 \%$ de jóvenes, el $91 \%$ de los adultos de tuvieron tallas entre 51 y $91 \mathrm{~mm} \mathrm{AC} \mathrm{(Fig.} \mathrm{2).} \mathrm{Ambas}$ poblaciones presentan características similares en la estructura de tallas donde la mayoría de los individuos son jóvenes y los individuos adultos mayores a $90 \mathrm{~mm}$ AC son escasos. Estos resultados difieren de los obtenidos por Molina-Ocampo (2001) y Nevárez-Martínez 

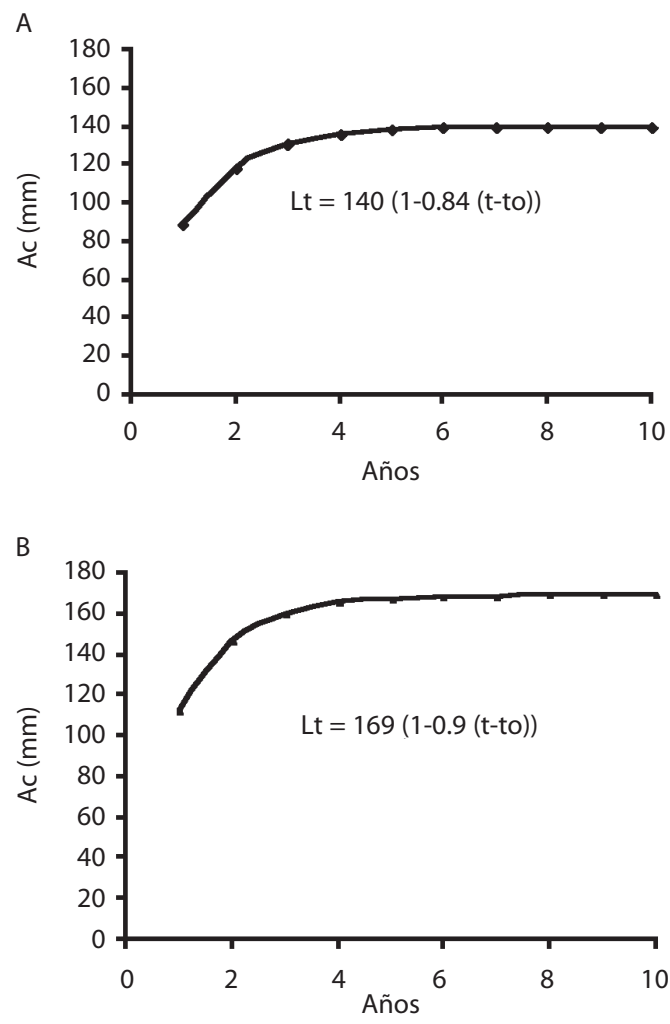

Fig. 5. Crecimiento estimado con el modelo von Bertalanffy: A. C. arcuatus y B. C. bellicosus.

Fig. 5. Growth predicted by the von Bertalanffy model: A. C. arcuatus, and B. C. bellicosus.

et al. (2003), donde la estructura de tallas fue de 20 a $98 \mathrm{~mm}$ de longitud de caparazón (LC) con medias entre $65 \mathrm{~mm}$ y $72 \mathrm{~mm}$ LC para ambas especies de jaibas, para fines comparativos $(\mathrm{LC}=0.5045 * \mathrm{AC})$ los resultados del presente estudio corresponden a 18 y $42 \mathrm{~mm}$ LC. La diferencia se atribuye a que los datos de Molina-Ocampo (2000) y Nevárez-Martínez et al. (2003), provienen de capturas comerciales de jaibas realizadas con trampas, y los datos del presente estudio provienen de recolectas con redes de arraste. En este sentido, la contribución de este estudio aporta información acerca de una parte de la población que no se había descrito previamente y que es consistente con la función ecológica de estos cuerpos de agua costeros de ser hábitat para reproducción y crecimiento de estas especies (Williams 1974), la recolección con red de arrastre permitió capturar individuos que se encuentran asociados a masas de macroalgas (Arreola-Lizarraga et al. 2003), antes de reclutarse al stock explotado; adicionalmente, este estudio muestra que las jaibas en esta laguna se encuentran maduras antes de alcanzar la talla de captura recomendada en el litoral del Pacífico mexicano que es de $95 \mathrm{~mm}$ AC para C. arcuatus y de $115 \mathrm{~mm} \mathrm{AC}$ para C. bellicosus (Ramírez-Félix et al. 2003).

Relación ancho-peso: La relación anchopeso mostró que en ambas especies los machos obtienen mayor talla y peso que las hembras. Nuestros resultados coincidieron con estudios realizados con las mismas especies en otras localidades del Pacífico mexicano (Paul 1982b, Molina-Ocampo 2001), así como con lo observado en otras especies del género Callinectes en la costa del Atlántico (Tagatz 1968, Olmi III y Bishop 1983, García-Montes et al. 1987). Esto en los braquiuros parece ser una generalidad asociada con el proceso reproductivo debido a que: (1) las hembras detienen su crecimiento para destinar gran parte de la energía a la reproducción, mientras que los machos continúan creciendo aún después de alcanzar la madurez sexual y (2) la cópula se realiza cuando la hembra está mudada y mientras regenera el exoesqueleto, el macho que la toma debe tener mayor tamaño para protegerla de depredadores (Sastry 1983).

Parámetros de crecimiento: En ambas especies de jaibas el crecimiento fue isométrico. Nevárez-Martínez et al. (2003) también observaron crecimiento isométrico en ambas especies de jaibas en las lagunas Las Guásimas y Lobos, México. Sin embargo, otros estudios de crecimiento en C. bellicosus (Molina-Ocampo 2001) y en C. arcuatus (Paul 1982b) mostraron crecimiento alométrico; en particular, Molina-Ocampo (2001) atribuyó el crecimiento alométrico a un desfasamiento en la condición biológica óptima de las hembras debido a bajas tasas de alimentación y mayor gasto energético en la temporada reproductiva; aunque esto es razonable, Nevárez-Martínez 
et al. (2003) indicaron que con ambos sexos o separándolos obtuvieron crecimiento isométrico. Cabe señalar que las bases de datos de Paul (1982b), Molina-Ocampo (2001) y NevárezMartínez et al. (2003) se construyeron a partir de capturas comerciales donde la población estuvo representada principalmente por machos adultos y la base de datos del presente estudio se construyó a partir de recolectas con red de arrastre donde la población estuvo representada principalmente por jóvenes. Considerando lo anterior y la coincidencia de los resultados de Nevárez-Martínez et al. (2003) con los obtenidos en este estudio es posible sugerir que ambas especies de jaibas tienden a tener un crecimiento isométrico que eventualmente puede tener un sesgo hacia el crecimiento alométrico debido a la presencia de hembras en la temporada reproductiva.

Los parámetros de $\mathrm{K}$ y $\mathrm{L} \infty$ estimados para cada especie, no indicaron disminución en la tasa de crecimiento durante el periodo de estudio. Esto lo atribuimos a que nuestros muestreos se dirigieron a capturar individuos de todas las tallas de la población, mientras que en capturas de pesca comercial existe una selectividad de tallas y la información proviene de individuos adultos.

El conocimiento de los parámetros de crecimiento, se puede aplicar para estimar el tamaño que alcanzarán los organismos hasta una talla máxima asintótica en un tiempo dado. Los parámetros de crecimiento estimados mostraron que la edad relativa a la cual los individuos alcanzan el máximo crecimiento es entre los tres y cuatro años de edad para ambas especies.

Los estudios previos al presente trabajo realizados en el Pacífico mexicano que tratan sobre crecimiento de C. arcuatus y C. bellicosus se basan en datos que provienen de las capturas comerciales con predominancia de individuos adultos (Paul 1982b, Molina-Ocampo 2001, Nevárez-Martínez et al. 2003), y este estudio aporta información fundamentalmente de jóvenes, el componente más importante de la población en el interior de las lagunas costeras. Al comparar los parámetros de crecimiento

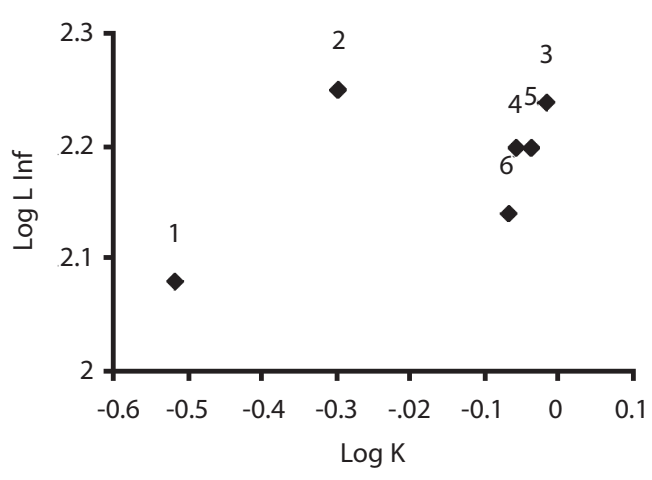

Fig. 6. Comparación de parámetros de crecimiento de $C$. arcuatus y $C$. bellicosus mediante el índice Fi prima ( $\left.\varnothing^{\prime}\right)$ de Munro (1-3 Escamilla-Montes 1998; 4 Molina 1999b; 5-6 este estudio).

Fig. 6. Comparison between growth parameters of $C$. arcuatus and $C$. bellicosus by Munro's index ( $\left.\varnothing^{\prime}\right)$ (1-3 Escamilla-Montes 1998; 4 Molina 1999b; 5-6 our study).

estimados en este estudio con los resultados en las contribuciones previas mediante el índice de la Fi prima de Munro Ø' (Munro y Pauly 1983) observamos que los valores se encuentran dentro de los intervalos conocidos para ambas especies (Fig. 6).

Concluimos que en la laguna Las Guásimas C. bellicosus es más abundante que $C$. arcuatus y que ambas poblaciones presentan una estructura de tallas similar y caracterizada, principalmente, por jóvenes entre $37-75 \mathrm{~mm}$ $\mathrm{AC}$ y adultos entre 76-90 mm AC; los machos crecen más que las hembras; los individuos tienen un crecimiento isométrico y alcanzan su máximo crecimiento entre los tres y cuatro años de edad.

\section{AGRADECIMIENTOS}

Agradecemos al personal técnico adscrito a los laboratorios de manejo costero y calidad del agua del CIBNOR, S. C. Unidad Sonora su apoyo en el trabajo de campo y laboratorio. El primer autor agradece al CONACyT la beca otorgada para realizar sus estudios de maestría. 


\section{RESUMEN}

Presentamos información sobre la estructura de tallas, la relación ancho-peso y los parámetros de crecimiento de las jaibas Callinectes arcuatus y C. bellicosus. Las muestras fueron recolectadas mensualmente con una red de arrastre tipo camaronera durante el día y la noche desde marzo 1998 hasta febrero 2000 en una laguna costera del Golfo de California. C. bellicosus $(\mathrm{n}=878)$ fue más abundante que C. arcuatus $(\mathrm{n}=357)$ y su intervalo de talla de 8.4-166 mm ancho del caparazón (AC) y 9-130 mm AC, respectivamente. Ambas poblaciones son representadas principalmente por jóvenes (75 mm AC) entre $37-75 \mathrm{~mm}$ AC y adultos entre 76-90 mm AC. La relación ancho-peso mostró que los machos crecen más que las hembras en ambas especies y se observó una tendencia de crecimiento isométrico. Los parámetros de crecimiento para C. arcuatus estimados con el modelo von Bertalanffy fueron: $\mathrm{K}=$ 0.84 año $^{-1}, \mathrm{~L} \infty=140$ a $-0.12 \mathrm{~mm}$ para $C$. arcuatus, y $\mathrm{K}=0.9$ año $^{-1}, \mathrm{~L} \infty=169$ a $-0.11 \mathrm{~mm}$ para $C$. bellicosus. Estos resultados mostraron que la edad relativa a la cual se alcanza el crecimiento máximo es entre los tres y cuatro años de edad para ambas especies.

Palabras clave: jaibas, crecimiento, estructura de tallas, Callinectes arcuatus, Callinectes bellicosus.

\section{REFERENCIAS}

Arreola-Lizárraga, J.A., L.G. Hernández-Moreno, S. Hernández-Vázquez, F.J. Flores-Verdugo, C. Lechuga-Devezé \& A. Ortega-Rubio. 2003. Ecology of Callinectes arcuatus and C. bellicosus (Decapoda: Portunidae) in a coastal lagoon of Northwest Mexico. Crustaceana 76: 651-664

Brusca, R. 1980. Common intertidal invertebrates of the Gulf of California. University of Arizona, Fénix, Arizona, EEUU. 513 p.

Dittel, A. \& C.E. Epifanio. 1984. Growth and development of the portunid crab Callinectes arcuatus Ordway: Zoeae, Megalopae and Jóvenes. J. Crust. Biol. 4: $491-494$.

García-Montes, J.F., A. Gracía \& L.A. Soto. 1987. Morfometría, crecimiento relativo y fecundidad de la jaiba del golfo Callinectes similis Williams, 1966 (Decapoda: Portunidae). Ciencias Marinas 13: 137161.

Hendrickx, M.E. 1995. Cangrejos, p. 565 - 636. In W. Fisher, F. Krupp, W. Schneider, C. Sommer, K.E. Carpenter \& V.H. Niem (eds.). Guía FAO para la identificación de especies para los fines de la pesca. Pacífico Centro-oriental. FAO, Roma, Italia.

Kjerfve, B. \& K.E. Magill. 1989. Geographic and hydrodynamic characteristics of shallow coastal lagoons. Marine Geology 88: 187-199.

Molina-Ocampo, R.E. 2001. Jaiba del Estado de Sonora Callinectes bellicosus, p. 329-348. In Sustentabilidad y Pesca Responsable en México. Evaluación y Manejo. INP/SEMARNAP, Sonora, México.

Munro, J.L. \& D. Pauly. 1983. A simple method for comparing growth of fishes and invertebrates. Fishbyte 1: 5-6.

Nevárez-Martinez, M.O., J. López-Martínez, C. CervantesValle, E. Miranda-Mier, R. Morales-Azpeitia \& M. L. Anguiano-Carrasco. 2003. Evaluación biológica y pesquera de las jaibas Callinectes bellicosus y Callinectes arcuatus (Brachyura: Decapada: Portunidae) en las bahía de Guásimas y Lobos, Sonora, México, p. 143-151. In M.E. Hendrickx (ed.). Contribuciones al Estudio de los Crustáceos del Pacífico Este 3 [Contributions to the Study of East Pacific Crustaceans 3]. Instituto de Ciencias del Mar y Limnología, UNAM, México.

Olmi III, E. \& J.M. Bishop. 1983. Variations in total width-weight relationships of blue crabs, Callinectes sapidus, in relation to sex, maturity, molt satage, and caparace form. J. Crust. Biol. 3: 575-581.

Paul, R.K.G. 1982a. Observation on the ecology and distribution of swimming crabs of the genus Callinectes (Decapoda: Brachyura: Portunidae) in the Gulf of California, Mexico. Crust. 42: 96-100.

Paul, R.K.G. 1982b. Abundance, breeding and growth of Callinectes arcuatus Ordway and Callinectes toxotes Ordway (Decapoda: Brachyura: Portunidae) in a lagoon system on the Mexican Pacific Coast. Estuar. Coast. Shelf Sci. 14: 13-26.

Pauly, D. 1980. On the interrelation between natural mortality, growth parameters and mean environmental temperature in 175 fish stock. Journal du conseil, Conseil International pour 1'Exploration de la Mer 39: $175-192$.

Rathbun, M. 1930. The cancroid crabs of America of the families Euryalidae, Portunidae, Atelecyclidae, Cancridae and Xanthidae. Bull. U.S. Nat. Mus. 152: $1-609$.

Ramírez-Félix, E., J. Singh-Cabanillas, H.A. Gil-López, S. Sarmiento-Náfate, I. Salazar, L.G. Montemayor, J.A. García-Borbón, G. Domínguez \& N. Castañeda. 
2003. La pesquería de jaiba (Callinectes spp.) en el Pacífico mexicano: diagnóstico y propuesta de regulación. Comisión Nacional de Acuacultura y Pesca (CONAPESCA), Instituto Nacional de la Pesca, México, 48 p.

Sastry, A.N. 1983. Ecological aspects of reproduction, p. 179-270. In D. E. Bliss (ed.). The biology of Crustacea. Academic, Nueva York, EEUU.
Sparre, P. \& C. Venema. 1995. Introducción a la evaluación de recursos pesqueros tropicales. FAO, Valparaíso, Chile. 420 p.

Tagatz, M.E. 1968. Biology of the blue crab Callinectes sapidus Rathbun in the St Johns river, Florida. Fish. Bull. 67: 17-33.

Williams, A.B. 1974. The swimming crabs of the genus Callinectes. Fisher. Bull. 72: 685-798. 
